\title{
Diabetes Management in Correctional Institutions
}

American Diabetes Association

A $\mathrm{t}$ any given time, over 2 million people are incarcerated in prisons and jails in the U.S (1). It is estimated that nearly 80,000 of these inmates have diabetes, a prevalence of $4.8 \%$ (2). In addition, many more people pass through the corrections system in a given year. In 1998 alone, over 11 million people were released from prison to the community (1). The current estimated prevalence of diabetes in correctional institutions is somewhat lower than the overall U.S. prevalence of diabetes, perhaps because the incarcerated population is younger than the general population. The prevalence of diabetes and its related comorbidities and complications, however, will continue to increase in the prison population as current sentencing guidelines continue to increase the number of aging prisoners and the incidence of diabetes in young people continues to increase.

People with diabetes in correctional facilities should receive care that meets national standards. Correctional institutions have unique circumstances that need to be considered so that all standards of care may be achieved (3). Correctional institutions should have written policies and procedures for the management of diabetes and for training of medical and correctional staff in diabetes care practices. These policies must take into consideration issues such as security needs, transfer from one facility to another, and access to medical personnel and equipment, so that all appropriate levels of care are provided. Ideally, these policies should encourage or at least allow patients to self-manage their diabetes. Ultimately, diabetes management is dependent upon having access to needed medical personnel and equipment. Ongoing diabetes therapy is important in order to reduce the risk of later complications, including cardiovascular events, visual loss, renal failure, and amputation. Early identification and intervention for people with diabetes is also likely to reduce short-term risks for acute complications requiring transfer out of the facility, thus improving security.

This document provides a general set of guidelines for diabetes care in correctional institutions. It is not designed to be a diabetes management manual. More detailed information on the management of diabetes and related disorders can be found in the American Diabetes Association (ADA) Clinical Practice Recommendations, published each year in January as the first supplement to Diabetes Care, as well as the "Standards of Medical Care in Diabetes" (4) contained therein. This discussion will focus on those areas where the care of people with diabetes in correctional facilities may differ, and specific recommendations are made at the end of each section.

\section{INTAKE MEDICAL ASSESSMENT}

\section{Reception screening}

Reception screening should emphasize patient safety. In particular, rapid identification of all insulin-treated persons with diabetes is essential in order to identify those at highest risk for hypo- and hyperglycemia and diabetic ketoacidosis (DKA). All insulin-treated patients should have a capillary blood glucose $(\mathrm{CBG})$ determination within 1-2 h of arrival. Signs and symptoms of hypo- or hyperglycemia can often be confused with intoxication or withdrawal from drugs or alcohol. Individuals with diabetes exhibiting signs and symptoms consistent with hypoglycemia, particularly altered mental status, agitation, combativeness, and diaphoresis, should have finger-stick blood glucose levels measured immediately.

\section{Intake screening}

Patients with a diagnosis of diabetes should have a complete medical history

Originally approved 1989. Most recent revision, 2008.

DOI: $10.2337 / \mathrm{dcl} 3-\mathrm{S} 086$

(C) 2013 by the American Diabetes Association. Readers may use this article as long as the work is properly cited, the use is educational and not for profit, and the work is not altered. See http://creativecommons.org/ licenses/by-nc-nd/3.0/ for details.

and physical examination by a licensed health care provider with prescriptive authority in a timely manner. If one is not available on site, one should be consulted by those performing reception screening. The purposes of this history and physical examination are to determine the type of diabetes, current therapy, alcohol use, and behavioral health issues, as well as to screen for the presence of diabetes-related complications. The evaluation should review the previous treatment and the past history of both glycemic control and diabetes complications. It is essential that medication and medical nutrition therapy (MNT) be continued without interruption upon entry into the correctional system, as a hiatus in either medication or appropriate nutrition may lead to either severe hypo- or hyperglycemia that can rapidly progress to irreversible complications, even death.

\section{Intake physical examination and laboratory}

All potential elements of the initial medical evaluation are included in Table 7 of the ADA's "Standards of Medical Care in Diabetes," referred to hereafter as the "Standards of Care" (4). The essential components of the initial history and physical examination are detailed in Fig. 1. Referrals should be made immediately if the patient with diabetes is pregnant.

\section{Recommendations}

- Patients with a diagnosis of diabetes should have a complete medical history and undergo an intake physical examination by a licensed health professional in a timely manner. (E)

- Insulin-treated patients should have a CBG determination within $1-2 \mathrm{~h}$ of arrival. (E)

- Medications and MNT should be continued without interruption upon entry into the correctional environment. (E)

\section{SCREENING FOR DIABETES-}

Consistent with the ADA Standards of Care, patients should be evaluated for diabetes risk factors at the intake physical and at appropriate times thereafter. Those who are at high risk should be considered for blood glucose screening. If pregnant, a risk assessment for gestational diabetes 
Within 1-2 hrs.

RECEPTION SCREENING

-Identify all inmates with diabetes currently using insulin therapy or at high risk for hypoglycemia

- ALL insulin-treated patients: screening CBG and urine ketone test (as clinically indicated)

- Any patient exhibiting signs/symptoms consistent with hypoglycemia: immediate CBG

-Continue usual meal schedule and medication administration

Within 2-24 hrs.

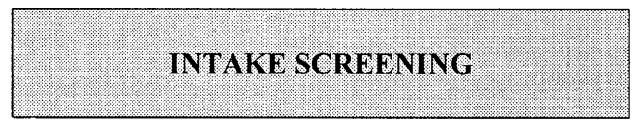

- Type and duration of diabetes

-Confirm current therapy

-Presence of complications

-Family history

-Pregnancy screen in all female patients

of childbearing age with diabetes
-Assess alcohol use

-Identify behavioral health issues

such as depression, distress, suicidal ideation

-Assess prior diabetes education

All subjects with diabetes should have physician evaluation. If no physician available, physician should be consulted.

Within 2 hrs. - 2 weeks
INTAKE PHYSICAI. EXAM

LABORATORY - COMPLICATIONS SCREENING

Complete exam including:

-Height, weight

-Blood pressure

-Eye (retinal) exam

- Cardiac

-Peripheral pulses

-Foot and neurologic exam
Laboratory studies:

-A1C and glucose

-Lipid Profile

-Microalbumin screen (Alb/Cr ratio)

-Urine ketones (as clinically indicated)

-AST/ALT (as clinically indicated)

-Creatinine (as clinically indicated)

Figure 1-Essential components of the initial history and physical examination. Alb/Cr ratio, albumin-to-creatinine ratio; ALT, alanine aminotransferase; AST, aspartate aminotransferase.

mellitus (GDM) should be undertaken at the first prenatal visit. Patients with clinical characteristics consistent with a high risk for GDM should undergo glucose testing as soon as possible. High-risk women not found to have GDM at the initial screening and average-risk women should be tested between 24 and 28 weeks of gestation. For more detailed information on screening for both type 2 and gestational diabetes, see the ADA Position Statement "Screening for Type 2 Diabetes" (5) and the Standards of Care (4).

MANACEMENT PLAN-Glycemic control is fundamental to the management of diabetes. A management plan to achieve normal or near-normal glycemia with an A1C goal of $<7 \%$ should be developed for diabetes management at the time of initial medical evaluation. Goals should be individualized (4), and less stringent treatment goals may be appropriate for patients with a history of severe hypoglycemia, patients with limited life expectancies, elderly adults, and individuals with comorbid conditions (4). This plan should be documented in the patient's record and communicated to all persons involved in his/her care, including security staff. Table 1, taken from the ADA Standards of Care, provides a summary of recommendations for setting glycemic control goals for adults with diabetes.

People with diabetes should ideally receive medical care from a physiciancoordinated team. Such teams include, but are not limited to, physicians, nurses, dietitians, and mental health professionals with expertise and a special interest in diabetes. It is essential in this collaborative and integrated team approach that individuals with diabetes assume as active a role in their care as possible. Diabetes self-management education is an integral component of care. Patient selfmanagement should be emphasized, and the plan should encourage the involvement of the patient in problem solving as much as possible.

It is helpful to house insulin-treated patients in a common unit, if this is possible, safe, and consistent with providing access to other programs at the correctional institution. Common housing not only can facilitate mealtimes and medication administration, but also po- tentially provides an opportunity for diabetes self-management education to be reinforced by fellow patients.

\section{NUTRITION AND FOOD}

SERVICES - Nutrition counseling and menu planning are an integral part of the multidisciplinary approach to diabetes management in correctional facilities. A combination of education, interdisciplinary communication, and monitoring food intake aids patients in understanding their medical nutritional needs and can facilitate diabetes control during and after incarceration.

Nutrition counseling for patients with diabetes is considered an essential component of diabetes self-management. People with diabetes should receive individualized MNT as needed to achieve treatment goals, preferably provided by a registered dietitian familiar with the components of MNT for persons with diabetes.

Educating the patient, individually or in a group setting, about how carbohydrates and food choices directly affect diabetes control is the first step in facilitating self-management. This education enables the patient to identify better food 
Table 1-Summary of recommendations for glycemic, blood pressure, and lipid control for most adults with diabetes

\begin{tabular}{ll}
\hline AlC & $<7.0 \% *$ \\
Blood pressure & $<140 / 80 \mathrm{mmHg} \dagger$ \\
Lipids & $<100 \mathrm{mg} / \mathrm{dL}$ \\
$\quad$ LDL cholesterol & $(<2.6 \mathrm{mmol} / \mathrm{L}) \neq$ \\
\hline
\end{tabular}

* More or less stringent glycemic goals may be ap propriate for individual patients. Goals should be individualized based on duration of diabetes, age/life expectancy, comorbid conditions, known CVD or advanced microvascular complications, hypoglycemia unawareness, individual and patient considerations $\dagger$ Based on patient characteristics and response to therapy, lower SBP targets may be appropriate. $¥$ In individuals with overt CVD, a lower LDL cholesterol goal of $<70 \mathrm{mg} / \mathrm{dL}(1.8 \mathrm{mmol} / \mathrm{L})$, using a high dose of a statin, is an option.

selections from those available in the dining hall and commissary. Such an approach is more realistic in a facility where the patient has the opportunity to make food choices.

The easiest and most cost-effective means to facilitate good outcomes in patients with diabetes is instituting a hearthealthy diet as the master menu (6). There should be consistent carbohydrate content at each meal, as well as a means to identify the carbohydrate content of each food selection. Providing carbohydrate content of food selections and/or providing education in assessing carbohydrate content enables patients to meet the requirements of their individual MNT goals. Commissaries should also help in dietary management by offering healthy choices and listing the carbohydrate content of foods.

The use of insulin or oral medications may necessitate snacks in order to avoid hypoglycemia. These snacks are a part of such patients' medical treatment plans and should be prescribed by medical staff.

Timing of meals and snacks must be coordinated with medication administration as needed to minimize the risk of hypoglycemia, as discussed more fully in the MEDICATION section of this document. For further information, see the ADA Position Statement "Nutrition Recommendations and Interventions for Diabetes" (7).

\section{URGENT AND EMERGENCY}

ISSUES - All patients must have access to prompt treatment of hypo- and hyperglycemia. Correctional staff should be trained in the recognition and treatment of hypo- and hyperglycemia, and appropriate staff should be trained to administer glucagon. After such emergency care, patients should be referred for appropri- ate medical care to minimize risk of future decompensation.

Institutions should implement a policy requiring staff to notify a physician of all CBG results outside of a specified range, as determined by the treating physician (e.g., $<50$ or $>350 \mathrm{mg} / \mathrm{dL}$ ).

\section{Hyperglycemia}

Severe hyperglycemia in a person with diabetes may be the result of intercurrent illness, missed or inadequate medication, or corticosteroid therapy. Correctional institutions should have systems in place to identify and refer to medical staff all patients with consistently elevated blood glucose as well as intercurrent illness.

The stress of illness in those with type 1 diabetes frequently aggravates glycemic control and necessitates more frequent monitoring of blood glucose (e.g., every 4-6 h). Marked hyperglycemia requires temporary adjustment of the treatment program and, if accompanied by ketosis, interaction with the diabetes care team. Adequate fluid and caloric intake must be ensured. Nausea or vomiting accompanied with hyperglycemia may indicate DKA, a life-threatening condition that requires immediate medical care to prevent complications and death. Correctional institutions should identify patients with type 1 diabetes who are at risk for DKA, particularly those with a prior history of frequent episodes of DKA. For further information see "Hyperglycemic Crisis in Diabetes" (8).

\section{Hypoglycemia}

Hypoglycemia is defined as a blood glucose level $<70 \mathrm{mg} / \mathrm{dL}$. Severe hypoglycemia is a medical emergency defined as hypoglycemia requiring assistance of a third party and is often associated with mental status changes that may include confusion, incoherence, combativeness, somnolence, lethargy, seizures, or coma. Signs and symptoms of severe hypoglycemia can be confused with intoxication or withdrawal. Individuals with diabetes exhibiting signs and symptoms consistent with hypoglycemia, particularly altered mental status, agitation, and diaphoresis, should have their CBG levels checked immediately.

Security staff who supervise patients at risk for hypoglycemia (i.e., those on insulin or oral hypoglycemic agents) should be educated in the emergency response protocol for recognition and treatment of hypoglycemia. Every attempt should be made to document CBG before treatment. Patients must have immediate access to glucose tablets or other glucosecontaining foods. Hypoglycemia can generally be treated by the patient with oral carbohydrates. If the patient cannot be relied on to keep hypoglycemia treatment on his/her person, staff members should have ready access to glucose tablets or equivalent. In general, 15-20 g oral glucose will be adequate to treat hypoglycemic events. CBG and treatment should be repeated at 15-min intervals until blood glucose levels return to normal (>70 mg/ dL).

Staff should have glucagon for intramuscular injection or glucose for intravenous infusion available to treat severe hypoglycemia without requiring transport of the hypoglycemic patient to an outside facility. Any episode of severe hypoglycemia or recurrent episodes of mild to moderate hypoglycemia require reevaluation of the diabetes management plan by the medical staff. In certain cases of unexplained or recurrent severe hypoglycemia, it may be appropriate to admit the patient to the medical unit for observation and stabilization of diabetes management.

Correctional institutions should have systems in place to identify the patients at greater risk for hypoglycemia (i.e., those on insulin or sulfonylurea therapy) and to ensure the early detection and treatment of hypoglycemia. If possible, patients at greater risk of severe hypoglycemia (e.g., those with a prior episode of severe hypoglycemia) may be housed in units closer to the medical unit in order to minimize delay in treatment.

\section{Recommendations}

- Train correctional staff in the recognition, treatment, and appropriate referral for hypo- and hyperglycemia. (E)

- Train appropriate staff to administer glucagon. (E)

- Train staff to recognize symptoms and signs of serious metabolic decompensation, and immediately refer the patient for appropriate medical care. (E)

- Institutions should implement a policy requiring staff to notify a physician of all CBG results outside of a specified range, as determined by the treating physician (e.g., $<50$ or $>350 \mathrm{mg} / \mathrm{dL}$ ). (E)

- Identify patients with type 1 diabetes who are at high risk for DKA. (E)

MEDICATION-Formularies should provide access to usual and customary 
oral medications and insulins necessary to treat diabetes and related conditions. While not every brand name of insulin and oral medication needs to be available, individual patient care requires access to short-, medium-, and long-acting insulins and the various classes of oral medications (e.g., insulin secretagogues, biguanides, $\alpha$-glucosidase inhibitors, and thiazolidinediones) necessary for current diabetes management.

Patients at all levels of custody should have access to medication at dosing frequencies that are consistent with their treatment plan and medical direction. If feasible and consistent with security concerns, patients on multiple doses of shortacting oral medications should be placed in a "keep on person" program. In other situations, patients should be permitted to self-inject insulin when consistent with security needs. Medical department nurses should determine whether patients have the necessary skill and responsible behavior to be allowed self-administration and the degree of supervision necessary. When needed, this skill should be a part of patient education. Reasonable syringe control systems should be established.

In the past, the recommendation that regular insulin be injected 30-45 min before meals presented a significant problem when "lock downs" or other disruptions to the normal schedule of meals and medications occurred. The use of multiple-dose insulin regimens using rapid-acting analogs can decrease the disruption caused by such changes in schedule. Correctional institutions should have systems in place to ensure that rapid-acting insulin analogs and oral agents are given immediately before meals if this is part of the patient's medical plan. It should be noted however that even modest delays in meal consumption with these agents can be associated with hypoglycemia. If consistent access to food within 10 min cannot be ensured, rapidacting insulin analogs and oral agents are approved for administration during or immediately after meals. Should circumstances arise that delay patient access to regular meals following medication administration, policies and procedures must be implemented to ensure the patient receives appropriate nutrition to prevent hypoglycemia.

Both continuous subcutaneous insulin infusion and multiple daily insulin injection therapy (consisting of three or more injections a day) can be effective means of implementing intensive diabetes management with the goal of achieving near-normal levels of blood glucose (9). While the use of these modalities may be difficult in correctional institutions, every effort should be made to continue multiple daily insulin injection or continuous subcutaneous insulin infusion in people who were using this therapy before incarceration or to institute these therapies as indicated in order to achieve blood glucose targets.

It is essential that transport of patients from jails or prisons to off-site appointments, such as medical visits or court appearances, does not cause significant disruption in medication or meal timing. Correctional institutions and police lockups should implement policies and procedures to diminish the risk of hypo- and hyperglycemia by, for example, providing carry-along meals and medication for patients traveling to off-site appointments or changing the insulin regimen for that day. The availability of prefilled insulin "pens" provides an alternative for off-site insulin delivery.

\section{Recommendations}

- Formularies should provide access to usual and customary oral medications and insulins to treat diabetes and related conditions. (E)

- Patients should have access to medication at dosing frequencies that are consistent with their treatment plan and medical direction. (E)

- Correctional institutions and police lock-ups should implement policies and procedures to diminish the risk of hypo- and hyperglycemia during offsite travel (e.g., court appearances). (E)

\section{ROUTINE SCREENING FOR AND MANAGEMENT OF DIABETES COMPLICATIONS-All patients} with a diagnosis of diabetes should receive routine screening for diabetes-related complications, as detailed in the ADA Standards of Care (4). Interval chronic disease clinics for persons with diabetes provide an efficient mechanism to monitor patients for complications of diabetes. In this way, appropriate referrals to consultant specialists, such as optometrists/ ophthalmologists, nephrologists, and cardiologists, can be made on an as-needed basis and interval laboratory testing can be done.

The following complications should be considered:
- Foot care: Recommendations for foot care for patients with diabetes and no history of an open foot lesion are described in the ADA Standards of Care. A comprehensive foot examination is recommended annually for all patients with diabetes to identify risk factors predictive of ulcers and amputations. Persons with an insensate foot, an open foot lesion, or a history of such a lesion should be referred for evaluation by an appropriate licensed health professional (e.g., podiatrist or vascular surgeon). Special shoes should be provided as recommended by licensed health professionals to aid healing of foot lesions and to prevent development of new lesions.

- Retinopathy: Annual retinal examinations by a licensed eye care professional should be performed for all patients with diabetes, as recommended in the ADA Standards of Care. Visual changes that cannot be accounted for by acute changes in glycemic control require prompt evaluation by an eye care professional.

- Nephropathy: An annual spot urine test for determination of microalbuminto-creatinine ratio should be performed. The use of ACE inhibitors or angiotensin receptor blockers is recommended for all patients with albuminuria. Blood pressure should be controlled to $<140 /$ $80 \mathrm{mmHg}$

- Cardiac: People with type 2 diabetes are at a particularly high risk of coronary artery disease. Cardiovascular disease (CVD) risk factor management is of demonstrated benefit in reducing this complication in patients with diabetes. Blood pressure should be measured at every routine diabetes visit. In adult patients, test for lipid disorders at least annually and as needed to achieve goals with treatment. Use aspirin therapy (75-162 mg/day) in all adult patients with diabetes and cardiovascular risk factors or known macrovascular disease. Current national standards for adults with diabetes call for treatment of lipids to goals of LDL $\leq 100$, HDL $>40$, triglycerides $<150 \mathrm{mg} / \mathrm{dL}$, and blood pressure to a level of $<140 / 80$ $\mathrm{mmHg}$

\section{MONITORING/TESTS OF}

CLYCEMIA-Monitoring of $\mathrm{CBG}$ is a strategy that allows caregivers and people with diabetes to evaluate diabetes management regimens. The frequency of monitoring will vary by patients' glycemic control and diabetes regimens. Patients 
with type 1 diabetes are at risk for hypoglycemia and should have their CBG monitored three or more times daily. $\mathrm{Pa}$ tients with type 2 diabetes on insulin need to monitor at least once daily and more frequently based on their medical plan. Patients treated with oral agents should have CBG monitored with sufficient frequency to facilitate the goals of glycemic control, assuming that there is a program for medical review of these data on an ongoing basis to drive changes in medications. Patients whose diabetes is poorly controlled or whose therapy is changing should have more frequent monitoring. Unexplained hyperglycemia in a patient with type 1 diabetes may suggest impending DKA, and monitoring of ketones should therefore be performed.

Glycated hemoglobin (A1C) is a measure of long-term (2- to 3-month) glycemic control. Perform the AlC test at least two times a year in patients who are meeting treatment goals (and who have stable glycemic control) and quarterly in patients whose therapy has changed or who are not meeting glycemic goals.

Discrepancies between CBG monitoring results and $\mathrm{AlC}$ may indicate a hemoglobinopathy, hemolysis, or need for evaluation of CBG monitoring technique and equipment or initiation of more frequent $\mathrm{CBG}$ monitoring to identify when glycemic excursions are occurring and which facet of the diabetes regimen is changing.

In the correctional setting, policies and procedures need to be developed and implemented regarding CBG monitoring that address the following:

- infection control

- education of staff and patients

- proper choice of meter

- disposal of testing lancets

- quality control programs

- access to health services

- size of the blood sample

- patient performance skills

- documentation and interpretation of test results

- availability of test results for the health care provider (10)

\section{Recommendations}

- In the correctional setting, policies and procedures need to be developed and implemented to enable CBG monitoring to occur at the frequency necessitated by the individual patient's glycemic control and diabetes regimen. (E)
- AlC should be checked every 3-6 months. (E)

\section{SELF-MANAGEMENT \\ EDUCATION-Self-management ed-} ucation is the cornerstone of treatment for all people with diabetes. The health staff must advocate for patients to participate in self-management as much as possible. Individuals with diabetes who learn self-management skills and make lifestyle changes can more effectively manage their diabetes and avoid or delay complications associated with diabetes. In the development of a diabetes selfmanagement education program in the correctional environment, the unique circumstances of the patient should be considered while still providing, to the greatest extent possible, the elements of the "National Standards for Diabetes Self-Management Education and Support" (11). A staged approach may be used depending on the needs assessment and the length of incarceration. Table 2 sets out the major components of diabetes self-management education. Survival skills should be addressed as soon as possible; other aspects of education may be provided as part of an ongoing education program.

Ideally, self-management education is coordinated by a certified diabetes educator who works with the facility to develop polices, procedures, and protocols to ensure that nationally recognized education guidelines are implemented. The educator is also able to identify patients who need diabetes self-management education, including an assessment of the patients' medical, social, and diabetes histories; diabetes knowledge, skills, and behaviors; and readiness to change.

STAFF EDUCATION-Policies and procedures should be implemented to ensure that the health care staff has adequate knowledge and skills to direct the management and education of persons with diabetes. The health care staff needs to be involved in the development of the correctional officers' training program. The staff education program should be at a lay level. Training should be offered at least biannually, and the curriculum should cover the following:

- what diabetes is

- signs and symptoms of diabetes
- risk factors

- signs and symptoms of, and emergency response to, hypo- and hyperglycemia

- glucose monitoring

- medications

- exercise

- nutrition issues including timing of meals and access to snacks

\section{Recommendations}

- Include diabetes in correctional staff education programs. (E)

ALCOHOL AND DRUGS-Patients with diabetes who are withdrawing from drugs and alcohol need special consideration. This issue particularly affects initial police custody and jails. At an intake facility, proper initial identification and assessment of these patients are critical. The presence of diabetes may complicate detoxification. Patients in need of complicated detoxification should be referred to a facility equipped to deal with highrisk detoxification. Patients with diabetes should be educated in the risks involved with smoking. All inmates should be advised not to smoke. Assistance in smoking cessation should be provided as practical.

\section{TRANSFER AND}

DISCHARGE-Patients in jails may be housed for a short period of time before being transferred or released, and it is not unusual for patients in prison to be transferred within the system several times during their incarceration. One of the many challenges that health care providers face working in the correctional system is how to best collect and communicate important health care information in a timely manner when a patient is in initial police custody, is jailed short term, or is transferred from facility to facility. The importance of this communication becomes critical when the patient has a chronic illness such as diabetes.

Transferring a patient with diabetes from one correctional facility to another requires a coordinated effort. To facilitate a thorough review of medical information and completion of a transfer summary, it is critical for custody personnel to provide medical staff with sufficient notice before movement of the patient.

Before the transfer, the health care staff should review the patient's medical record and complete a medical transfer summary that includes the patient's 
Table 2-Major components of diabetes self-management education

Survival skills
- hypo-/hyperglycemia
- sick day management
- medication
- monitoring
- foot care

Daily management issues

- disease process

- physical activity

- medications

- monitoring
- nutritional management

- acute complications

- risk reduction

- goal setting/problem solving

- psychosocial adjustment

- preconception care/pregnancy/gestational diabetes management

current health care issues. At a minimum, the summary should include the following:

- the patient's current medication schedule and dosages

- the date and time of the last medication administration

- any recent monitoring results (e.g., CBG and AlC)

- other factors that indicate a need for immediate treatment or management at the receiving facility (e.g., recent episodes of hypoglycemia, history of severe hypoglycemia or frequent DKA, concurrent illnesses, presence of diabetes complications)

- information on scheduled treatment/ appointments if the receiving facility is responsible for transporting the patient to that appointment

- name and telephone/fax number of a contact person at the transferring facility who can provide additional information, if needed

The medical transfer summary, which acts as a quick medical reference for the receiving facility, should be transferred along with the patient. To supplement the flow of information and to increase the probability that medications are correctly identified at the receiving institution, sending institutions are encouraged to provide each patient with a medication card to be carried by the patient that contains information concerning diagnoses, medication names, dosages, and frequency. Diabetes supplies, including diabetes medication, should accompany the patient.

The sending facility must be mindful of the transfer time in order to provide the patient with medication and food if needed. The transfer summary or medical record should be reviewed by a health care provider upon arrival at the receiving institution.

Planning for patients' discharge from prisons should include instruction in the long-term complications of diabetes, the necessary lifestyle changes and examinations required to prevent these complications, and, if possible, where patients may obtain regular follow-up medical care. A quarterly meeting to educate patients with upcoming discharges about community resources can be valuable. Inviting community agencies to speak at these meetings and/or provide written materials can help strengthen the community link for patients discharging from correctional facilities.

Discharge planning for the patients with diabetes should begin 1 month before discharge. During this time, application for appropriate entitlements should be initiated. Any gaps in the patient's knowledge of diabetes care need to be identified and addressed. It is helpful if the patient is given a directory or list of community resources and if an appointment for follow-up care with a community provider is made. A supply of medication adequate to last until the first postrelease medical appointment should be provided to the patient upon release. The patient should be provided with a written summary of his/her current health care issues, including medications and doses, recent A1C values, etc.

\section{Recommendations}

- For all interinstitutional transfers, complete a medical transfer summary to be transferred with the patient. (E)

- Diabetes supplies and medication should accompany the patient during transfer. (E)

- Begin discharge planning with adequate lead time to insure continuity of care and facilitate entry into community diabetes care. (E)

\section{SHARING OF MEDICAL INFORMATION AND}

RECORDS-Practical considerations may prohibit obtaining medical records from providers who treated the patient before arrest. Intake facilities should implement policies that 1) define the circumstances under which prior medical records are obtained (e.g., for patients who have an extensive history of treatment for complications); 2) identify person(s) responsible for contacting the prior provider; and 3) establish procedures for tracking requests.

Facilities that use outside medical providers should implement policies and procedures for ensuring that key information (e.g., test results, diagnoses, physicians' orders, appointment dates) is received from the provider and incorporated into the patient's medical chart after each outside appointment. The procedure should include, at a minimum, a means to highlight when key information has not been received and designation of a person responsible for contacting the outside provider for this information.

All medical charts should contain CBG test results in a specified, readily accessible section and should be reviewed on a regular basis.

\section{CHILDREN AND ADOLESCENTS WITH DIABETES-Children and} adolescents with diabetes present special problems in disease management, even outside the setting of a correctional institution. Children and adolescents with diabetes should have initial and follow-up care with physicians who are experienced in their care. Confinement increases the difficulty in managing diabetes in children and adolescents, as it does in adults with diabetes. Correctional authorities also have different legal obligations for children and adolescents.

\section{Nutrition and activity}

Growing children and adolescents have greater caloric/nutritional needs than adults. The provision of an adequate amount of calories and nutrients for adolescents is critical to maintaining good nutritional status. Physical activity should be provided at the same time each day. If increased physical activity occurs, additional CBG monitoring is necessary and additional carbohydrate snacks may be required. 
Medical management and follow-up Children and adolescents who are incarcerated for extended periods should have follow-up visits at least every 3 months with individuals who are experienced in the care of children and adolescents with diabetes. Thyroid function tests and fasting lipid and microalbumin measurements should be performed according to recognized standards for children and adolescents (12) in order to monitor for autoimmune thyroid disease and complications and comorbidities of diabetes.

Children and adolescents with diabetes exhibiting unusual behavior should have their CBG checked at that time. Because children and adolescents are reported to have higher rates of nocturnal hypoglycemia (13), consideration should be given regarding the use of episodic overnight blood glucose monitoring in these patients. In particular, this should be considered in children and adolescents who have recently had their overnight insulin dose changed.

PRECNANCY-Pregnancy in a woman with diabetes is by definition a high-risk pregnancy. Every effort should be made to ensure that treatment of the pregnant woman with diabetes meets accepted standards $(14,15)$. It should be noted that glycemic standards are more stringent, the details of dietary management are more complex and exacting, insulin is the only antidiabetic agent approved for use in pregnancy, and a number of medications used in the management of diabetic comorbidities are known to be teratogenic and must be discontinued in the setting of pregnancy.

\section{SUMMARY AND KEY}

POINTS-People with diabetes should receive care that meets national standards. Being incarcerated does not change these standards. Patients must have access to medication and nutrition needed to manage their disease. In patients who do not meet treatment targets, medical and behavioral plans should be adjusted by health care professionals in collaboration with the prison staff. It is critical for correctional institutions to identify particularly high-risk patients in need of more intensive evaluation and therapy, including pregnant women, patients with advanced complications, a history of repeated severe hypoglycemia, or recurrent DKA.

A comprehensive, multidisciplinary approach to the care of people with diabetes can be an effective mechanism to improve overall health and delay or prevent the acute and chronic complications of this disease.

Acknowledgments - The following members of the American Diabetes Association/National Commission on Correctional Health Care Joint Working Group on Diabetes Guidelines for Correctional Institutions contributed to the revision of this document: Daniel L. Lorber, MD, FACP, CDE (chair); R. Scott Chavez, MPA, PA-C; Joanne Dorman, RN, CDE, CCHP-A; Lynda K. Fisher, MD; Stephanie Guerken, RD, CDE; Linda B. Haas, CDE, RN; Joan V. Hill, CDE, RD; David Kendall, MD; Michael Puisis, DO; Kathy Salomone, CDE, MSW, APRN; Ronald M. Shansky, MD, MPH; and Barbara Wakeen, $\mathrm{RD}, \mathrm{LD}$.

\section{References}

1. National Commission on Correctional Health Care: The Health Status of Soon-toBe Released Inmates: A Report to Congress. Vol. 1. Chicago, NCCHC, 2002

2. Hornung CA, Greifinger RB, Gadre S: A Projection Model of the Prevalence of Selected Chronic Diseases in the Inmate Population. Vol. 2. Chicago, NCCHC, 2002, p. $39-56$

3. Puisis M: Challenges of improving quality in the correctional setting. In Clinical Practice in Correctional Medicine. St. Louis, MO, Mosby-Yearbook, 1998, p. 16-18

4. American Diabetes Association: Standards of medical care in diabetes-2013 (Position Statement). Diabetes Care 36 (Suppl. 1): S11-S66, 2013

5. American Diabetes Association: Screening for type 2 diabetes (Position Statement). Diabetes Care 27 (Suppl. 1):S11-S14, 2004
6. Krauss RM, Eckel RH, Howard B, Appel LJ, Daniels SR, Deckelbaum RJ, Erdman JW Jr, Kris-Etherton P, Goldberg IJ, Kotchen TA, Lichtenstein AH, Mitch WE, Mullis R, Robinson K, Wylie-Rosett J, St Jeor S, Suttie J, Tribble DL, Bazzarre TL: American Heart Association Dietary Guidelines: revision 2000: a statement for healthcare professionals from the Nutrition Committee of the American Heart Association. Stroke 31:2751-2766, 2000

7. American Diabetes Association: Nutrition recommendations and interventions for diabetes (Position Statement). Diabetes Care 31 (Suppl. 1):S61-S78, 2008

8. American Diabetes Association: Hyperglycemic crisis in diabetes (Position Statement). Diabetes Care 27 (Suppl. 1): S94-S102, 2004

9. American Diabetes Association: Continuous subcutaneous insulin infusion (Position Statement). Diabetes Care 27 (Suppl. 1):S110, 2004

10. American Diabetes Association: Tests of glycemia in diabetes (Position Statement). Diabetes Care 27 (Suppl. 1):S91-S93, 2004

11. Haas L, Maryniuk M, Beck J, Cox CE, Duker P, Edwards L, Fisher EB, Hanson L, Kent D, Kolb L, McLaughlin S, Orzeck E, Piette JD, Rhinehart AS, Rothman R, Sklaroff S, Tomky D, Youssef G, on behalf of the 2012 Standards Revision Task Force: National standards for diabetes self-management education and support. Diabetes Care 36 (Suppl. 1):S100-S108, 2013

12. International Society for Pediatric and Adolescent Diabetes: Consensus Guidelines 2000: ISPAD Consensus Guidelines for the Management of Type 1 Diabetes Mellitus in Children and Adolescents. Zeist, Netherlands, Medical Forum International, 2000, p. 116,118

13. Kaufman FR, Austin J, Neinstein A, Jeng L, Halyorson M, Devoe DJ, Pitukcheewanont P: Nocturnal hypoglycemia detected with the continuous glucose monitoring system in pediatric patients with type 1 diabetes. J Pediatr 141:625-630, 2002

14. American Diabetes Association: Gestational diabetes mellitus (Position Statement). Diabetes Care 27 (Suppl. 1):S88-S90, 2004

15. Jovanovic L (Ed.): Medical Management of Pregnancy Complicated by Diabetes. 4th ed. Alexandria, VA, American Diabetes Association, 2009 